\title{
The Research on WebIDE of Integrated Development Environment Oriented Eye Operating System
}

\author{
http://dx.doi.org/10.3991/ijoe.v9i4.2936 \\ DENG Zhenrong, ZHANG Chuan*, HUANG Wenming, and FAN Fei \\ Guilin University of Electronic Technology, Guilin, China
}

\begin{abstract}
Online operating system based on $\mathrm{B} / \mathrm{S}$ structure, WebOS become a precedent for advancing the development of the Web4.0 era. This article mainly elaborated the basic concept and future prospects of the online operating system eyeOS which introduced by the Europeans, and the design principle and idea is analyzed, followed by analysis of several major factors restricting eyeOS. And by using the architecture of server, extended to its function, put forward the researching and design method of WebIDE of integrated development environment oriented eye operating system.
\end{abstract}

Index Terms-EyeOS; WebIDE; Cloud Computing; Web4.0

\section{Introduction}

Cloud computing is a hot technical terminology in recent years, many experts assert that cloud computing can change the technical basis of Internet, and industrial pattern would be affected. Many large enterprises have joined the research of cloud computing technology and Cloud-based services. The goal of cloud computing is that, based on the distributed system, it is now bringing high extensibility, availability and high-performance for users. Recently, a new definition to cloud computing was presented by NIST[1](National Institute of Standards and Technology), the definition is that cloud computing is a ubiquitous, to provide convenient and on-demand access to the sharing model of configuration of network resource which include network tools, storage space, application and server resource etc. This definition of cloud computing structure includes three aspects: SaaS (software as a service), PaaS (platform as a service), IaaS (infrastructure as a service). With the development of cloud computing, the technology of networks is turning from Web1.0 (e.g. portals) into Web4.0 (e.g. web operating system) era.

Cloud computing based on the internet business calculation model provides a very good platform for the thin client application. The eyeOS based on the concept of web operating system is the trend of a new cyber source application. eyeOS makes full use of the characteristics of desktop virtualization, and it subverts the concept based on traditional local physical mechanism of resource management, client applications. This method lets people anywhere use their own desktop environment. With the development of software as a service (SaaS) cloud computing application level structure in the network application, eyeOS provides efficient support for it. The hybrid desktop virtualization model will become the future development trend of Web technology.

\section{SySTEM FRAMEWORK AND FILE SYSTEM OF EYEOS}

\section{A. The Concept of EyeOS}

The eyeOS based on browser is virtual operation system, to provide customers with similar local desktop interface. In order to let software developers develop network desktop applications as well as the development of desktop applications, eyeOS provides a set of tools for application development. eyeOS application development using the universal graphics library, allows JavaScript developers to use high level controls, such as windows, buttons, list etc, without the need to focus on the underlying processing. eyeOS provides a solution of resource storage control, permission assignment and user account management by means of the web interface. In fact, the real sense of webOS has not developed, because of the present hardware and technical conditions, can only be called web-desktop. eyeOS based on the $\mathrm{B} / \mathrm{S}$ of framework, just download the eyeOS file server and deployed on the local server can be easily run. eyeOS based on plug-in extension is maintain by all over the world in the open source community, it has own FTP, browsers, word processors and other applications. It is to make full use of its open source advantage, can finish the development of applications on the platform.

\section{B. The System Framework of EyeOS}

Function component of the eyeOS system mainly include two parts: the JavaScript and the PHP, and running on the Apache server, which has many functions can be used by the JavaScript libraries, through it, the development will become more simple. Similarly, the server also has php class library and API interface. As shown in Fig.1, the application is divided into two parts: one part running on the client (browser), another part running on a network server. The user interface of running on the client uses JavaScript, when do some operations, such as reading a database, you need to run a php function to start the APIs that is provided by the server. 


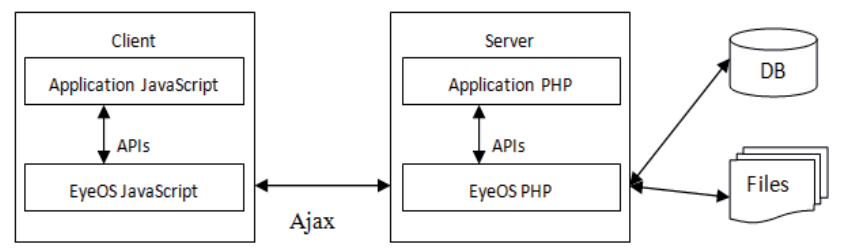

Figure 1. The system framework of eyeOS

In the eyeOS, all the components are modular, which means that the eyeOS system is composed of a series of components, such as user management. This modular structure do not have to worry about the back-end database. It can easily develop a new application or service without changing other components. In this case, there is no clear answer when someone asked what eyeOS data storage. In fact, everything depends on the application components(services) the type of database. Be default, the eyeOS is using mysql, but it can easily use oracle or sqlserver, even other database engine.

\section{The File System of EyeOS}

File storage and operation structure of eyeOS use the hierarchical model of unified, As shown in Fig.2, the file structure mainly includes index.php,setting.php configuration and system directory.

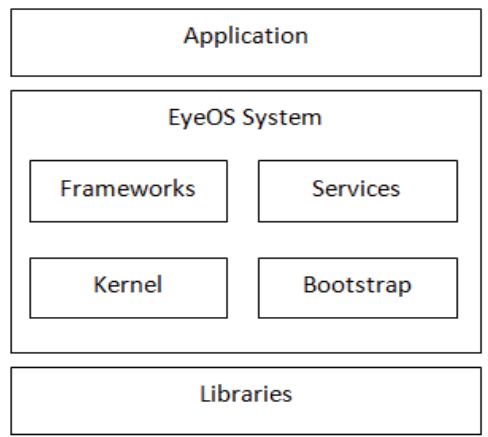

Figure 2. The file structure of eyeOS

eyeOS directory is called "eye-root", contains the whole the structure of eyeOS and user's data. There is an internal file called called. htaccess in this directory, to prevent directly access to the system directory from the browser, so it can only access the root directory of eyeOS by loading a page of index.php. The directory tree of eyeOS contains the following documents:

- Apps: This dirrectory contains the desktop of eyeOS and a series directories of applicatio.

- Extern: Only the index.php is accessible from the outside, eyeOS must provide some methods to make the browser can load JavaScript, CSS and images etc. All the necessary resources must be placed in the external directory or its subfolders. When index.php receives a request like "index.php?extern=image.jpg", the contents of image.jpg within the extern directory is returned to the browser so that it can be loader.

- Extras: All of the eyeOS installation and configuration files and scripts are here in this directory, but need not the operation of the eyeOS system.

- System: The file system directory contains all of the system components.
- Bootstrap: This file is responsible for running some operations before loading the entire system. When running the application, ensure that all components, frameworks and service can be successfully loaded.

- Framework: The framework is the highest level of the components of the system, i.e, they are the most close to the application layer and the further away from the underlying details.

- Libraries: The library is responsible for providing support and security components for eyeOS, but they are not eyeOS from the perspectives of the server, just provide the support and guarantee in a relatively low level, ensure that the operating environment right.

- Servers: The service component is essential to the operation of eyeOS, because it manages the internal operation of the component. For example, a user management component will be become a service, and a zip extract component must not because of system components, and therefore cannot be called a service. Many of the service will be discussed separately, or discuss deeply. For example, process management and user management are components of eyeOS. All applications can interact through the APIs.

- Kernel: The kernel is the component that manages the other components. Because eyeOS is a web application, the kernel is not like one in an operation system, but the kernel is the core component that manages the implementation of the remaining components that form the engine of eyeOS.

- Test: The Test directory contains a unit test.

- User: In the users directory, there is one subdirectory for each user. This sub-directory contains the user's files including documents and setting types of files.

- Workgroups: The workgroup directory contains the working groups of users.

\section{The Problem of The DeVElopMent of EyeOS}

\section{A. Processing Speed and Transmission Bandwidth}

The eyeOS based on ajax is a web application, it sent the customer's request through the asynchronous transmission. If you use eyeOS to process the documents or other work, may feel very slow, investigate its reason, is generally not computer configuration, but the network delay. When using a strong performance, complex of application, need to maintain high-speed communication between the client and the server, because a good environment of bandwidth will directly affect the development of eyeOS.

\section{B. Stable Service and Data Security}

eyeOS is based on a cloud platform, along with the performance of cloud computing, to promote the development of IT industry, its safety problems gradually exposed. The confidentiality, integrity, real-time requirements of data must get further attention in the cloud computing environment. Perhaps with the concept of the private cloud, restricting the development of eyeOS will got a good solution. 


\section{Extended Free Functions}

A new software can attract users to experience and use, there must be adequate functions, so the development of eyeOS need more support.

\section{APPLICATION BASED ON EYEOS}

\section{A. The Concept of WebIDE}

Current software development, especially the development of Web, for many beginners, bottleneck contains the following parts: the selection and building of development environment and the deployment of applications. Even skilled developers or small and medium-sized enterprises, in the face of the development and deployment of short period project, will also be pestered beyond endurance, the most important thing is that the repetitive and tedious work will delay the development progress and increase development costs. Is there a plan makes the individual developers or small workshop-type businesses can put aside these boring work instead of putting more energy into the actual development process. Therefore, proposing the WebIDE solution, it can integrate code management, compilation, execution(for many web applications may not have this step)and the final publish application in a web system.

\section{B. The Existing Products}

In the existing products, there is a similar example is AppFlower, but it has two shortcomings for the general developers:

- Limited to WebApps: AppFlower can be developed based on Html or Php.

- Freedom is too small: AppFlower provides a high degree of integration of the code editor or code generation tool for developers. But the user cannot choose and match their development environment, which to a large extent, limit developers play. The development of small Web Apps is an advantage for the monotonous, but if as a universal development tool is clearly inappropriate.

As can be seen, a web based on the sufficient application development environment is not possible achieved by the simple browser editor the backstage management of code, the receptionist must have developed greatly, the best solution is in the browser through the simulation (or real) basic ability of an operating system to build.

But the real significance of webOS has been developed, the ideal products such as Cloud Me (http://cloudme.com) and eyeOS (http://eyeos.org) and only be called web desktop. Considering the current level of technology and hardware conditions, the ideal solution is a compromise: Taking the web desktop as the foundation, all work such as code management, compilation and execution can be done on the server or cloud platform. In this way, the rough framework of webIDE system is outlined.

\section{The Design of System Structure}

There is a simple uml in the design of the system, as shown in Fig.3 and Fig4. The usecase graph mainly identifies the relationship between users and the usecase of webIDE and workflow, which is including the operations such as input, edit and background management of files. The static structure of webIDE is shown in the class graph, especially the internal structure of class and the relationship of class.

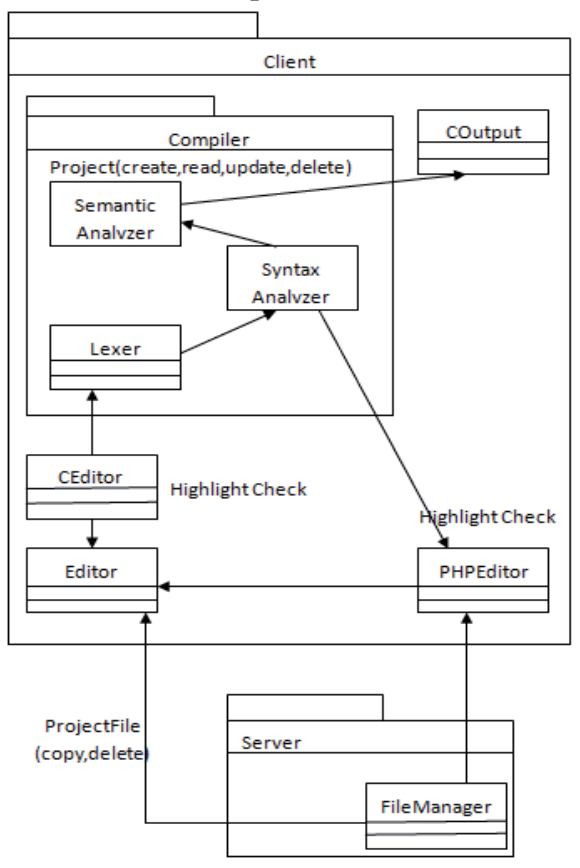

Figure 3. System structure of class graph

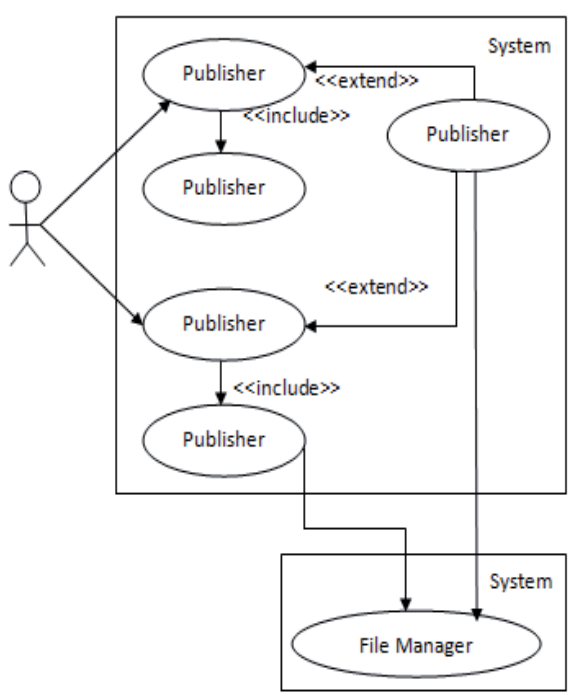

Figure 4. System structure of usecase graph

\section{Service Processing of WebIDE}

In the traditional network services, it is difficult to achieve Qos[9](Quality of Service) standard, but it is easy to get high performance and security service in the eyeOS which is based on cloud platform. Because the shared service is always deployed in the same cloud platform, namely eyeOS. In order to describe the process of webIDE service, the scenes description can be made, as shown in Fig.5. Assume that $U=\left\{\mathrm{u}_{1}, \mathrm{u}_{2}, \ldots, \mathrm{u}_{\mathrm{i}}\right\}$ represents a group of webIDE users, also known as cloud users, $\mathrm{T}=\left\{\mathrm{t}_{1}, \mathrm{t}_{2}, \ldots, \mathrm{t}_{\mathrm{i}}\right\}$ represents the request of a group of users, $\mathrm{t}=\{$ read,create,edit,...\} represents the actions of request, $R_{j}(j=1,2, \ldots, i)$ represents a set of results after the tasks submitted. Finally, the user's results can be summarized 
from different cloud service providers, namely $\mathrm{R}_{\mathrm{i}}=\left\{\mathrm{U}_{\mathrm{i}}, \mathrm{T}_{\mathrm{i}}\right\}$.

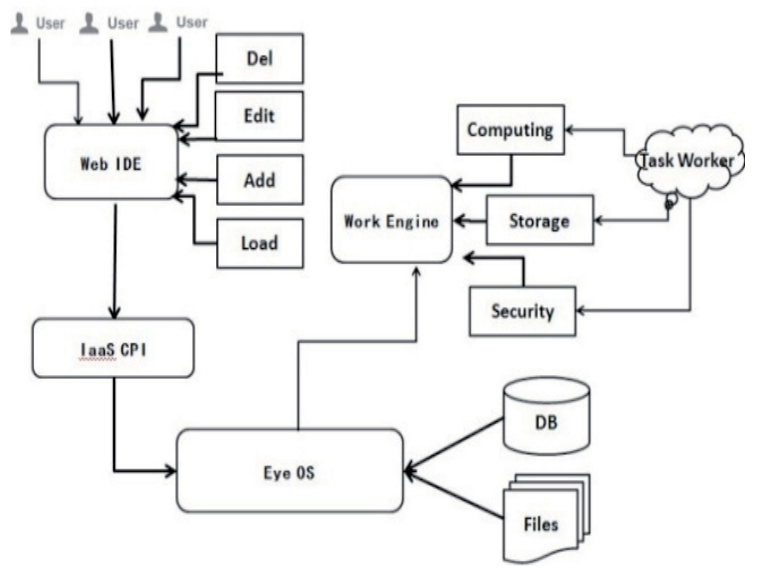

Figure 5. The service processing of webIDE

The state of webIDE is shown in Fig.6.

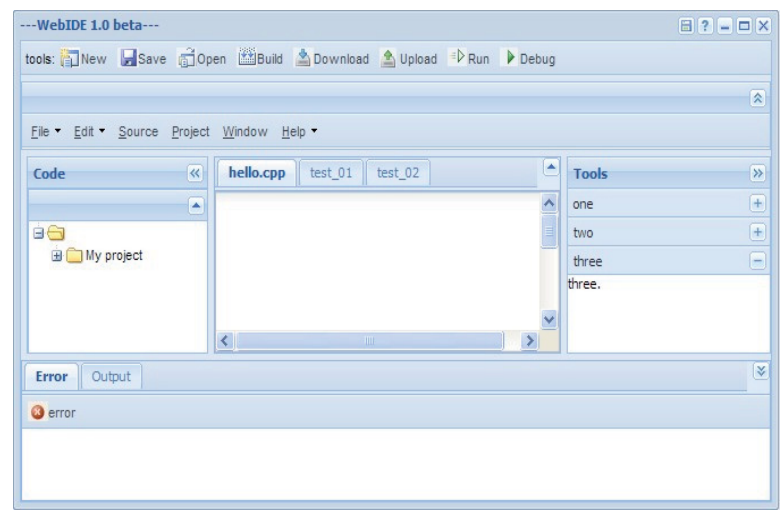

Figure 6. The service processing of WebIDE

\section{WEBIDE SUPPORT FOR WEB SERVICE}

Web service is an application component, which is logical for other applications to provide data and services. Each applications accesses the web service through some standard data format of net protocols and regulations such as http, xml and soap (Simple Object Access Protocol).A simple request to any complicated business processing can be executed by web service. Once deployed, other web application can discover and use web service. In order to webIDE away from the eyeOS platform, implementation of trans-regional, services of crossing platform and data sharing, webIDE based on the eyeOS can achieve service of web service through soap and xml functions.

\section{CONCLUSION}

A reasonable project needs to make a detailed analysis of the demand and the design process. The eyeOS is a new concept, and the $\mathrm{B} / \mathrm{S}$ architecture is the main direction of the development of software. The design of the webIDE preliminary achieve the goal of code editing, file management etc. Through the research and design, have reached the goal of the transformation of a IDE schema from $\mathrm{C} / \mathrm{S}$ architecture to $\mathrm{B} / \mathrm{S}$. With the continuous development of the network, the emergence of cloud computing, it will go far and become real product in the future.

Technical characteristics:

Break through the traditional model of $\mathrm{C} / \mathrm{S}$ structure of the IDE compiler, using eyeOS as foundation, development architecture for B/S IDE.

To solve problems, such as the development environment in the development of the project selection and construction as well as the final application deployment.

Programming integrated development environment (IDE), allows the user to write code, debug, deployment and application in the browser, suitable to replace the traditional desktop IDE software.

Technology improvement and future trend:

The compatibility of system and browser needs improvement.

Along with the rapid development of network time and cloud computing, the future of the webIDE to take full advantage of the cloud, file management, debug as well as the background related to handle all realize network, has reached the goal of $\mathrm{B} / \mathrm{S}$ architecture online integrated development environment.

Function remain to be improved, such as, tools of the project, including the use of simulation of public libraries and frameworks, server debugging environment, version control, coordination of many people, the realization of a high-performance, full-featured online integrated development environment.

\section{ACKNOWLEDGMENT}

This work is supported by the Science Research and Technology Development Projects of Guilin (No: 20120104-5), by the Postgraduate's Innovation Project of Guilin University of Electronic Technology(Grant No:XJY2012017), by the Nature Science Foundation of Guangxi (Grant No:2013GXNSFAA019350).

\section{REFERENCES}

[1] Y. Ren, B. Guan. " Research on eyeOS.”, Journal of Computer Science, 6 (2008): 164-167.

[2] Q. Ji, W.Tian,Y. Chen. "Construction of NixOS WebOS system based on AJAX Technology.", Journal of Computer of Engineering and Design, 5 (2008): 1198-1200.

[3] H. Wei. "Dynamic WebService framework system based on XML norms.", Journal of Computer Science, 6 (2004): 68-72.

[4] Bhaskar Prasad Rimal, Admela Jukan, Dimitrios Katsaros, Yves Goeleven. " Architectural Requirements for Cloud Computing Systems:An Enterprise Cloud Approach.”, Journal of Grid Computing, 9 (2011): 3-26. http://dx.doi.org/10.1007/s10723-0109171-y

[5] Nick BassIliades, Dimosthenis Anagnostopoulos, Ioannis Vlanavas. "Web Service composition Using a Deductive XML Rule Language.", Journal of Distributed and Parallel Databases, 17 (2005): 135-178. http://dx.doi.org/10.1007/s10619-004-0087-z

[6] S. Wang, Z. Liu. " Towards an accurate evaluation of quality of cloud service inservice-oriented cloud computing.", Journal of Intelligent Manufacturing, 2011.

[7] Jose Aguilar, Niriaska Perozo. “ Arichitecture of a Web Operating System Based on Multiagent Systems.”, Springer Berlin Heidelberg, 3687 (2005): 700-706.

[8] Nick Antonopoulos, Bhumip khasnabish. " Cloud Computing, Networking, and Services.", Journal of Network and Systems Management, 40 (2012): 463-467.

[9] Amit Sheth, Jorge Cardoso. " QoS for Service-oriented Middleware.", 6th World Multiconference on Systemics, 2012. 


\section{AUTHORS}

DENG Zhenrong, born in 1977, M.S, associate professor, Her main research interests include software architecture technology and protocol security. She is with School of Computer Science and Engineering, Guilin University of Electronic Technology, Guilin, Guangxi, China (e-mail: zhrdeng@guet.edu.cn).

ZHANG Chuan, born in 1988, M.S candidate, His main research interests include cloud computing, data mining, machine learning, and software engineering. He is now is with School of Computer Science and Engineering, Guilin University of Electronic Technology, Guilin, Guangxi, China (e-mail: noobzc@gmail.com).

HUANG Wenming, professor, His main research include graphics and image processing, software engineering, and information safety. He is with School of Computer Science and Engineering, Guilin University of
Electronic Technology, Guilin, Guangxi, China (e-mail: wmhuang@guet.edu .cn).

FAN Fei, born in 1988 , M.S candidate, His main research interests include workflow, cloud computing and image processing. He is now is with School of Computer Science and Engineering, Guilin University of Electronic Technology, Guilin, Guangxi, China (email:ffchinajoy@163.com).

This article is an extended and modified version of a paper presented at the 2012 International conference on Applied Science and Engineering Innovation (ASEI2012), held in Beijing, China, December 2012. This work was supported by the Science Research and Technology Development Projects of Guilin under Grant 20120104-5, by the Postgraduate's Innovation Project of Guilin University of Electronic Technology under Grant XJY2012017, by the Nature Science Foundation of Guangxi under Grant 2013GXNSFAA019350. Manuscript received 16 June 2013. Published as submitted by the authors 16 June 2013. 